\section{Desigualdade, pobreza e condições de saúde e nutrição na infância no Nordeste brasileiro}

\author{
Inequality, poverty, and childhood health and \\ nutritional conditions in Northeast Brazil
}

1 Escola de Nutrição, Universidade Federal da Bahia, Salvador Brasil.

2 Instituto de Saúde Coletiva, Universidade Federal da Bahia, Salvador, Brasil.

Correspondencia A. M. O. Assis Escola de Nutrição Universidade Federal da Bahia.

Rua Araújo Pinho 32 Salvador, $B A$ 40110-150, Brasil. amos@ufba.br

\section{Abstract}

This cross-sectional study analyzes the relationship between gradients of social inequalities and the household environment and health and nutritional conditions among 2,001 preschool children in ten counties (municipalities) in the State of Bahia, Brazil. The analysis used multinomial multivariate logistic regression. Children in the middle and lower tertiles on the poverty scale had significant and increasing odds of living in a household headed by a woman, an unemployed father, a mother with $\leq$ years of schooling, more than one child sharing the same bed, severe stunting, and retinol consumption below the median as compared to those in the upper tertile. More than one child in the home, weight deficit, and lipid consumption below the median were also significantly associated with the poorest tertile. Specific emergency health policies and measures must be implemented to minimize the burden imposed by poverty and social inequalities on childhood health and nutrition.

Social Inequity; Socioeconomic Factors; Child Welfare
Ana Marlúcia O. Assis ${ }^{1}$

Maurício L. Barreto 2

Nedja Silva Santos 1

Lucivalda Pereira Magalhães de Oliveira 1

Sandra Maria Chaves dos Santos 1

Sandra Maria Conceição Pinheiro 1

\section{Introdução}

O ambiente social e econômico em que vive a criança e sua família tem sido reconhecido como importante preditor das condições de saúde e nutrição na infância. As precárias condições de vida normalmente se expressam na baixa renda da unidade familiar, no limitado poder de compra, particularmente dos alimentos; nas precárias condições de saneamento do domicílio e do meio ambiente 1 ; e no limitado e desigual acesso a serviços de saúde com capacidade resolutiva 2 . Essas adversidades imprimem um padrão de morbidade na infância, caracterizado por altas taxas de doenças infecciosas, parasitárias e carenciais, particularmente a desnutrição protéica calórica e as carências específicas de micronutrientes 3 .

Nas últimas décadas o Brasil experienciou substancial melhora dos indicadores de saúde e nutrição 4, em especial na infância. No entanto, a melhora dos indicadores não se faz sentir de forma homogênea intra e entre as grandes regiões geográficas do país. A taxa da mortalidade infantil foi estimada em 34 mortes por mil nascidos vivos no ano 2000 para todo o país, mas distribuiu-se de forma heterogênea, com seus extremos nas regiões Nordeste $(50,1$ por mil nascidos vivos) e Sul (24,1 por mil nascidos vivos) 5 . Essas diferenças entre as taxas de mortalidade são ainda mais expressivas quando é considerado o nível de escolaridade materna, de tal forma 
que aumenta para o Brasil como um todo nos estratos de menor escolaridade e diminui naqueles de maior escolaridade materna; com diferencial mais robusto para a Região Nordeste 5 .

Informações disponíveis indicam também que a ocorrência da desnutrição em menores de cinco anos declinou em todo o país no período de 1986 a 1996, registrando-se redução de aproximadamente $51,7 \%$ para os déficits linear grave e moderado avaliados pelo indicador antropométrico altura/idade, e de $12 \%$ para o déficit ponderal medido pelo indicador peso/idade. Em que pese esse declínio, nota-se que as crianças do Nordeste brasileiro detêm os mais elevados déficits do indicador altura/idade $(17,9 \%)$ e peso/idade $(8,3 \%)$ quando comparadas com aqueles calculados para as crianças da Região Sul, respectivamente de $5,1 \%$ e $2 \%$, uma das regiões mais ricas do país ${ }^{6}$. Tendência de distribuição diferenciada segundo as áreas geográficas do país, é também registrada por pesquisa recente do Instituto Brasileiro de Geografia e Estatística (IGBE) 7, que identificou maior percentual de déficit de peso/idade para as crianças da área rural do Nordeste brasileiro $(8,7 \%)$ quando comparadas com a prevalência deste déficit entre as crianças do Sul total $(5,2 \%)$ e do Sul rural $(5,6 \%)$. Infelizmente nessas estimativas não estão disponíveis o déficit linear.

Independentemente da distribuição diferenciada da ocorrência da desnutrição na infância no Brasil, o declínio da prevalência observado ao longo das décadas é expressivo. E, é creditado à ampliação da cobertura vacinal, ao manejo adequado da diarréia e ao incentivo da prática do aleitamento materno 5 . No entanto, outros constrangedores ainda continuam a comprometer de forma expressiva o crescimento na infância, impedindo o pleno acesso desses serviços e/ou a aquisição de outros bens favoráveis à manutenção adequada do estado de saúde e nutrição. Essas condições podem explicar os resultados recentes do Ministério da Saúde, que indicam tendência da manutenção das altas prevalências de déficit no crescimento linear $(15,1 \%)$ e ponderal $(10,7 \%)$ entre as crianças menores de sete anos de idade do Programa Bolsa Alimentação no Nordeste do Brasil, oriundas de famílias expostas às mais precárias condições de vida; e sustentam também a manutenção do diferencial da distribuição dos déficits do crescimento na infância entre as regiões 8 .

Compõe ainda o cenário da desigualdade entre as regiões do país o acesso diferenciado ao saneamento básico, aos serviços de saúde e ao teto salarial 5 . Esse quadro caracteriza a situação de desigualdade inter-regional e pode-se pressupor a sua reprodução também dentro da pró- pria região, conformando um intenso gradiente diferencial no acesso a serviços e no consumo alimentar que pode se expressar em desigualdades em saúde.

Sem dúvida, um dos problemas que se coloca para a mensuração e compreensão dos determinantes das desigualdades em saúde, é dado pelo seu caráter plural e pela sobreposição de um quadro de múltiplas privações no acesso e utilização de bens sociais, a maioria derivada de desigualdades estruturais 9 .

A compreensão de como essas desigualdades conformam o quadro de saúde, particularmente na infância, é fundamental para formulação, implementação e avaliação de políticas públicas, uma vez que para situações desiguais, se fazem necessárias intervenções diferenciadas. Os indicadores de avaliação dessas intervenções podem exigir, também, parâmetros distintos que considerem a distância a ser percorrida na direção da eqüidade social e em saúde ${ }^{9}$. Nesse sentido, importa desvendar não só a existência das desigualdades em si, mas, sobretudo compreender seus fatores determinantes de forma a contribuir para a proposição e execução de políticas e programas capazes de intervir com efetividade sobre esse evento.

Assim, objetiva-se avaliar a associação entre os diferentes gradientes das condições sociais e econômicas e o estado de saúde e nutrição e fatores associados entre pré-escolares de municípios baianos, gerando evidências sobre a mediação da desigualdade como constrangedora do adequado estado nutricional na infância e direcionando a discussão sobre as situações concretas que limitam a eqüidade em saúde e nutrição de crianças em um espaço delimitado do nordeste brasileiro.

\section{Metodologia}

\section{População e amostra}

A amostra deste estudo é composta por 2001 préescolares oriundos de uma investigação mais ampla ${ }^{10}$, realizada no período de março de 1999 a março de 2000, envolvendo 1.887 domicílios das áreas urbana e rural de dez municípios do Estado da Bahia. A seleção desses municípios ocorreu por conveniência, sendo sorteados dentre aqueles com extensão territorial menor de $500 \mathrm{~km}^{2}$ que possuíssem de 10 a 15 mil habitantes e apresentassem maiores taxas de urbanização. Os critérios adotados para a seleção desses municípios foram definidos com base em questões logísticas que tornavam o estudo mais factível. No entanto, buscou-se resguardar a representação das dife- 
rentes áreas geográficas, com o objetivo de contemplar as diversidades sociais e econômicas do Estado da Bahia 10.

Os domicílios da área rural foram sorteados com base no agrupamento dos domicílios registrados nas fichas de coleta da contagem populacional de 1991, disponibilizadas pelo escritório do IBGE em Salvador 11. Aqueles da área urbana foram sorteados valendo-se da mesma base de dados. Todos os domicílios identificados foram visitados e todas as crianças menores de cinco anos de idade, residentes nestes domicílios, foram incluídas na amostra. Para a localização do domicílio sorteado e da família, contou-se com a ajuda dos agentes comunitários de saúde dos municípios.

Para o cálculo amostral adotou-se a prevalência do déficit do indicador peso/idade (8,3\%) e da altura/idade $(17,9 \%)$ da Região Nordeste, fornecida pela Pesquisa Nacional de Demografia e Saúde (PNDS) 6, tomando-se como base o número de crianças $<5$ anos de idade de cada município fornecido pelo Censo Demográfico de 1991 11. Assim, estimou-se o quantitativo de 2.733 pré-escolares, considerando-se a possibilidade de perda de $5 \%$. Optou-se por uma amostra aleatória estratificada, com alocação proporcional segundo a zona de residência das crianças (rural e urbana). As informações não estavam disponíveis para 85 crianças e, efetivamente, 2.648 integram a amostra do estudo original.

Para a avaliação do consumo alimentar optou-se por sortear uma criança por domicílio, dado que a variabilidade deste consumo para o grupo etário investigado é baixa $12 \mathrm{e}$, nessas crianças procedeu-se também a dosagem de hemoglobina. Com isso alcançou-se uma amostra de 2.009 crianças; para oito delas nem todas as informações estavam completas, conformando assim, a amostra final de 2.001 crianças. Considerando que essa amostra não foi estimada para avaliar a relação entre desigualdades sociais e econômicas e condições de saúde e nutrição, procedeu-se o cálculo do poder amostral, identificando-se poder de 90\% com nível de significância estatística $(\alpha)$ de 0,05 para avaliar a referida relação. Mesmo quando são adicionadas as 18 variáveis independentes no modelo, conforme o plano de análise deste estudo, esse poder de acerto permanece, condição que é satisfeita com um mínimo de 1.150 crianças na amostra 13.

\section{Coleta de dados}

Para a aferição do peso utilizou-se balança eletrônica (modelo E-150/3P, Filizola, São Paulo, Brasil) com capacidade para $100 \mathrm{~kg}$ e precisão de $100 \mathrm{~g}$. As crianças foram pesadas despidas ou usando calcinha ou cueca. A medição do comprimento das crianças menores de 24 meses e a altura daquelas maiores foi processada, respectivamente, por infantômetro de madeira e por estadiômetro (Leicester Height Measure, Londres, Inglaterra), ambos com precisão de $0,1 \mathrm{~cm}$. O peso e a altura foram tomados em duplicata e a média entre as medições foi adotada como medida final 14 . A padronização das medidas foi realizada de acordo com as recomendações técnicas 15 .

O sangue foi obtido por punção digital utilizando-se material descartável. A concentração de hemoglobina foi avaliada pelo método cianometahemoglobina, utilizando-se o sistema HemoCue 16. Quando o valor da hemoglobina era menor do que $9 \mathrm{~g} / \mathrm{dl}$ realizou-se uma segunda dosagem e a média entre as duas medições foi adotada como definitiva 16 .

O consumo alimentar foi avaliado pelo método recordatório de 24 horas. Com o intuito de contribuir com o entrevistado a recordar o tamanho das porções dos alimentos servidos e assim, minimizar o viés de informação, comum em estudo que versam sobre consumo alimentar, utilizou-se um álbum com desenhos de alimentos nas suas dimensões normais e medidas padrão para os líquidos 17 .

Os dados referentes ao ambiente do domicílio, sócio-econômicos e da criança foram obtidos utilizando-se questionários estruturados. Todas as informações foram fornecidas pela mãe ou responsável pela criança. A data de nascimento da criança foi comprovada por certidão de nascimento.

\section{Tratamento e definição das variáveis}

A variável dependente deste estudo é representada pelo índice sócio-econômico adaptado dos modelos propostos por Issler \& Giugliani 18 e Alvarez et al. 19, composto pela renda familiar per capita, posse de bens e utensílios domésticos, condições de moradia (material predominante da cobertura, parede e piso; condição de posse do domicílio e número de habitante por dormitório), saneamento domiciliar (origem da água usada no domicílio, disponibilidade de sanitário, de energia elétrica e esgotamento sanitário) e peri-domiciliar (destino do lixo). No cálculo desse índice, cada condição incorporada recebeu uma pontuação crescente de 0 a 4, com o valor zero caracterizando a situação menos favorável. Posteriormente esse índice foi estratificado em tercil, de tal forma que as crianças e suas famílias foram alocadas em três grupos, a saber: 1 o tercil (reúne 1/3 dos mais pobres), 2o tercil (intermediário, integra $1 / 3$ dos menos pobres) e 3o tercil (contempla $1 / 3$ dos mais ricos), este último to- 
mado como referência e os demais como riscos crescentes.

Adotou-se um conjunto de 18 variáveis independentes, selecionadas segundo a capacidade de se relacionar com o acesso e atenção à saúde, com os fatores biológicos (idade materna, paterna e da criança), relato de morbidade nos 15 dias anteriores à entrevista e internamento por qualquer causa nos 12 meses que antecederam a entrevista e com o estado nutricional e o consumo alimentar.

Os indicadores antropométricos altura/idade e peso/idade em $\mathrm{Z}$ escore caracterizam o estado antropométrico da criança. Com base nesses indicadores a criança foi classificada como desnutrida moderada/grave $(<-2 \mathrm{Z}$ escore), desnutrida leve ( $<-1$ a -2$)$ e eutrófica ( $>-1 \mathrm{Z}$ escore) tomando como referência o padrão Nacional Center for Health Statistics (NCHS) ${ }^{20}$. Adotou-se essa estratificação por compreender que a desnutrição de intensidade leve também tem importância epidemiológica no quadro de morbimortalidade na infância 21 .

Níveis de hemoglobina menores do que $11 \mathrm{~g} / \mathrm{dl}$ classificam o estado da anemia em criança de 6 a 59 meses de idade 16. Esse mesmo ponto de corte, utilizado na prática clínica, foi adotado para caracterizar a anemia em crianças menores de seis meses de idade, pela inexistência de recomendação para esta faixa etária 22 .

A prevalência foi adotada para descrever as características biológicas, demográficas, sociais e econômicas das famílias, assim como as condições de acesso ao serviço de saúde, da morbidade, do estado antropométrico e dos níveis de hemoglobina das crianças investigadas, segundo tercis de condições de vida.

Para a análise das associações de interesse utilizou-se a abordagem multinomial - uma extensão da regressão logística - que permite considerar a categorização da variável dependente em três níveis ${ }^{23}$. Inicialmente realizou-se a análise bivariada com o propósito de selecionar as variáveis para a composição do modelo multivariado. Para a seleção dessas variáveis optouse pelo nível de significância expresso por um p valor menor que $20 \%$ e para a permanência da variável no modelo final adotou-se o $\mathrm{p}$ valor menor que $5 \%$.

A análise do consumo alimentar foi realizada utilizando-se do programa Virtual Nutri (Philippi ST, Szarfarc SC, Laterza CR. Departamento de Nutrição, Faculdade de Saúde Pública, Universidade de São Paulo, São Paulo, Brasil). Os valores apurados para macro e micronutrientes de cada criança foram convertidos em medianas, por melhor expressar a distribuição no grupo estudado, e foram categorizados em dois estratos: acima (0) e abaixo (1) deste parâmetro estatístico. A categorização das demais variáveis encontrase apresentada de forma auto-explicativa nas tabelas de resultados.

Utilizou-se o programa Stata (Stata Corp., College Station, Estados Unidos) para a realização dos procedimentos estatísticos, por permitir ponderar as análises pelo efeito do conglomerado. $\mathrm{O}$ ajuste do modelo foi avaliado utilizando-se o teste de Wald 24 .

\section{Aspectos éticos}

A pertinência ética deste estudo foi atestada pelo Comitê de Ética do Hospital Professor Edgar Santos (HUPES), da Universidade Federal da Bahia. Atendendo aos pressupostos éticos que regem as pesquisas com seres humanos, as crianças portadoras de desnutrição e/ou anemia foram encaminhadas aos serviços de saúde para os cuidados médicos necessários.

\section{Resultados}

A distribuição percentual das características biológicas, nutricionais e de saúde das crianças, segundo os tercis de condições de vida, está apresentada na Tabela 1. Observou-se incremento na prevalência do déficit tanto ponderal quanto linear, nas formas leve e moderada/grave, à proporção que as crianças passavam do tercil mais rico para os mais pobres. Tendência similar, porém menos acentuada, foi observada para a ocorrência da anemia. Observou-se o aumento das prevalências dos indicadores do consumo alimentar abaixo da mediana do grupo à proporção que a criança se deslocava do tercil mais rico para o mais pobre.

A prevalência de morbidade referida nos 15 dias anteriores à entrevista e de internamento no ano anterior, bem como aquelas referentes ao sexo e à idade da criança distribuíram-se de forma similar entre os tercis de condições de vida.

Observou-se que a maior proporção das crianças do tercil mais pobre residia na área rural. Nesse mesmo patamar de condições de vida a chefia da residência era exercida majoritariamente pela mulher, o desemprego paterno atingiu o nível mais alto, a escolaridade materna era a mais baixa, o compartilhamento da cama pela criança com outra pessoa era comum e a existência de outra criança menor de cinco anos de idade no domicílio era mais freqüente (Tabela 2).

Os resultados das análises da regressão logística multinomial univariada (Tabela 3) indicaram chances crescentes para a maioria dos agravos nutricionais à proporção que as condições materiais 
de vida da criança iam se tornando mais precárias. A exceção foi observada somente para a anemia, que mostrou odds ratio (OR) similar segundo o estrato de condições de vida das crianças.

Notou-se, também, que a inadequação do peso e da altura em relação à idade, de caráter leve e moderado/grave, era evento freqüente entre as crianças do tercil de vida intermediário e entre as mais pobres, quando comparada com aquela de 1/3 das crianças mais ricas; mas o gradiente da inadequação era mais elevado para as mais pobres.

Identificou-se também que a criança, cuja condição de vida a classifica de mais pobre, tinha chance significantemente mais elevada de adoecimento nos 15 dias que precederam à entrevista, enquanto a chance de internamento nos últimos 12 meses da data da entrevista era mais elevada e estatisticamente significante para aquela do tercil intermediário, quando comparada com a mais rica.

As variáveis relacionadas ao consumo alimentar se associaram significantemente com as condições de vida das crianças. Quando a criança passava do tercil intermediário para o mais pobre aumentava a chance da caloria da dieta e do consumo de macro (proteína e lipídeo) e de micronutrientes (retinol e ferro) situar-se abaixo

\section{Tabela}

Distribuição percentual das características biológicas, do estado nutricional e de saúde das crianças segundo os tercis de condições sociais e econômicas em municípios baianos $(\mathrm{N}=2.001)$.

\begin{tabular}{|c|c|c|c|c|c|c|c|}
\hline \multirow[t]{2}{*}{ Variável } & \multicolumn{2}{|c|}{$\begin{array}{c}\text { 1o tercil } \\
\text { (mais pobre) }\end{array}$} & \multicolumn{2}{|c|}{$\begin{array}{c}\text { 2o tercil } \\
\text { (intermediário) }\end{array}$} & \multicolumn{2}{|c|}{$\begin{array}{l}3^{\circ} \text { tercil } \\
\text { (mais rico) }\end{array}$} & \multirow[t]{2}{*}{$p$ valor } \\
\hline & $\mathrm{n}$ & $\%$ & $\mathrm{n}$ & $\%$ & $n$ & $\%$ & \\
\hline \multicolumn{8}{|c|}{ Adequação ponderal (peso/idade) } \\
\hline Eutrofia & 398 & 56,7 & 427 & 66,0 & 503 & 77,6 & \\
\hline Déficit leve & 237 & 32,2 & 161 & 25,7 & 129 & 20,0 & \\
\hline Déficit moderado/grave & 80 & 11,1 & 50 & 8,1 & 16 & 2,4 & $<0,001$ \\
\hline \multicolumn{8}{|c|}{ Adequação linear (altura/idade) } \\
\hline Eutrofia & 368 & 53,2 & 399 & 61,9 & 502 & 76,3 & \\
\hline Déficit leve & 239 & 32,8 & 178 & 27,9 & 122 & 19,7 & \\
\hline Déficit moderado/grave & 108 & 14,0 & 61 & 10,2 & 24 & 4,0 & $<0,001$ \\
\hline \multicolumn{8}{|l|}{ Anemia } \\
\hline Não & 350 & 49,3 & 302 & 47,8 & 381 & 59,3 & \\
\hline $\operatorname{Sim}$ & 365 & 50,7 & 336 & 52,2 & 267 & 40,7 & $<0,001$ \\
\hline \multicolumn{8}{|l|}{ Sexo da criança } \\
\hline Masculino & 368 & 51,4 & 309 & 47,9 & 331 & 51,3 & \\
\hline Feminino & 347 & 48,6 & 329 & 52,1 & 317 & 48,7 & 0,3739 \\
\hline \multicolumn{8}{|l|}{ Idade da criança (meses) } \\
\hline$<6$ & 98 & 12,9 & 75 & 11,0 & 79 & 12,2 & \\
\hline $6-12$ & 79 & 11,1 & 75 & 11,6 & 69 & 10,4 & \\
\hline $12-24$ & 160 & 22,6 & 144 & 23,1 & 133 & 21,0 & \\
\hline$\geq 24$ & 378 & 53,4 & 344 & 54,3 & 367 & 56,5 & 0,8441 \\
\hline \multicolumn{8}{|c|}{ Referência a problema de doença * } \\
\hline Não & 246 & 33,8 & 237 & 37,9 & 249 & 39,2 & \\
\hline $\operatorname{Sim}$ & 469 & 66,2 & 401 & 62,1 & 399 & 60,8 & 0,124 \\
\hline \multicolumn{8}{|l|}{ História de internamento ** } \\
\hline $\operatorname{Sim}$ & 543 & 74,7 & 456 & 71,7 & 501 & 77,1 & \\
\hline Não & 169 & 25,3 & 177 & 28,3 & 145 & 22,9 & 0.096 \\
\hline \multicolumn{8}{|c|}{ Referência ao uso de fortificante $* \star \star$} \\
\hline Usou & 108 & 15,8 & 157 & 24,4 & 203 & 30,7 & \\
\hline Não usou & 607 & 84,2 & 481 & 75,6 & 445 & 69,3 & $<0,001$ \\
\hline \multicolumn{8}{|c|}{ Disponibilidade de caloria da dieta } \\
\hline Acima da mediana & 264 & 39,0 & 324 & 51,1 & 414 & 65,0 & \\
\hline Abaixo da mediana & 451 & 61,0 & 314 & 48,9 & 234 & 35,0 & $<0,001$ \\
\hline
\end{tabular}

(continua) 


\begin{tabular}{|c|c|c|c|c|c|c|c|}
\hline \multirow[t]{2}{*}{ Variável } & \multicolumn{2}{|c|}{$\begin{array}{c}\text { 10 tercil } \\
\text { (mais pobre) }\end{array}$} & \multicolumn{2}{|c|}{$\begin{array}{c}\text { 2o tercil } \\
\text { (intermediário) }\end{array}$} & \multicolumn{2}{|c|}{$\begin{array}{l}\text { 3o tercil } \\
\text { (mais rico) }\end{array}$} & \multirow[t]{2}{*}{$\mathrm{p}$ valor } \\
\hline & $\mathrm{n}$ & $\%$ & $\mathrm{n}$ & $\%$ & $\mathrm{n}$ & $\%$ & \\
\hline \multicolumn{8}{|l|}{ Consumo de proteína } \\
\hline Acima da mediana & 274 & 40,0 & 319 & 50,6 & 412 & 64,4 & \\
\hline Abaixo da mediana & 441 & 60,0 & 319 & 49,4 & 236 & 35,6 & $<0,001$ \\
\hline \multicolumn{8}{|l|}{ Consumo de lipídeo } \\
\hline Acima da mediana & 255 & 37,7 & 327 & 51,6 & 421 & 66,4 & \\
\hline Abaixo da mediana & 460 & 62,3 & 311 & 48,4 & 227 & 33,6 & $<0,001$ \\
\hline \multicolumn{8}{|l|}{ Consumo de retinol } \\
\hline Acima da mediana & 264 & 37,8 & 317 & 48,3 & 418 & 64,2 & \\
\hline Abaixo da mediana & 451 & 62,2 & 321 & 51,7 & 230 & 35,8 & $<0,001$ \\
\hline \multicolumn{8}{|l|}{ Consumo de ferro } \\
\hline Acima da mediana & 301 & 42,6 & 314 & 49,8 & 388 & 61,0 & \\
\hline Abaixo da mediana & 414 & 57,4 & 324 & 50,2 & 260 & 40,0 & $<0,001$ \\
\hline \multicolumn{8}{|l|}{ Consumo de zinco } \\
\hline Acima da mediana & 322 & 45,8 & 326 & 51,4 & 353 & 54,8 & \\
\hline Abaixo da mediana & 393 & 54,2 & 312 & 48,6 & 295 & 45,2 & 0,006 \\
\hline
\end{tabular}

* Nos 15 dias anteriores à entrevista;

** No último ano antes da entrevista;

*** Últimos 30 dias.

Nota: prevalência de desnutrição leve $(25,8 \%)$ e moderada/grave $(7,2 \%)$ avaliada pelo indicador peso/idade, e leve (26,6\%) e moderada/grave $(9,3 \%)$ segundo o indicador altura/idade e prevalência da anemia na amostra (47,7\%); razão de prevalência (RP) do déficit do indicador peso/idade entre crianças pobres e mais ricas ( $\mathrm{RP}=1,758$; IC95\%: 1,498-2,063) e altura/idade ( $\mathrm{RP}=1,983$; IC95\%: 1,690-2,328).

da mediana do grupo. Para o zinco a associação só foi observada para as crianças alocadas no tercil mais pobre.

Os fatores do ambiente domiciliar associaram-se também com as condições sociais e econômicas da família (Tabela 4). Nesse particular, a chance de a criança viver na área rural, da residência ser chefiada por uma mulher, de a mãe ter 40 anos ou mais e não ter concluído a 4 a serie do ensino fundamental, além de dormir com mais uma pessoa na mesma cama, ter mais de um irmão menor de cinco anos de idade e não usar fortificante no mês anterior à entrevista era menor entre as crianças do tercil intermediário e elevava-se consistentemente entre aquelas dos tercis mais pobres. Identificou-se também que o cuidado materno prestado à criança associouse negativamente com as condições de vida expressas nos tercis intermediário e no mais pobre, quando comparados com aqueles das crianças mais ricas.

Os resultados das análises de regressão logística multinomial multivariada (Tabela 5), indicaram que as chances de inadequação das condições nutricionais, de saúde e do ambiente se tornaram crescentes à proporção que a criança se deslocava do tercil mais rico para os mais pobres. Assim, as condições adversas do ambiente social e econômico mostraram maior gradiente de chance para as crianças do tercil intermediário seguidas por aquelas do tercil mais pobre, a exemplo de viver na área rural $(\mathrm{OR}=2,83$; IC95\%: 2,06-3,89, para o tercil intermediário e OR = 9,13; IC95\%: 6,47-12,90, para as mais pobres), ter a chefia da residência exercida por uma mulher $(\mathrm{OR}=1,52$; IC95\%: 1,09-2,12, para o tercil intermediário e OR = 1,88; IC95\%: 1,292,76 , para as mais pobres), ter pai na condição de desempregado (OR = 2,96; IC95\%: 2,19-4,00, para o tercil intermediário e OR $=4,90$; IC95\%: $3,47-6,92$, para $1 / 3$ mais pobre), a mãe não ter concluído a $4^{\text {a }}$ série do ensino fundamental (OR = 4,01; IC95\%: 3,05-5,27, para o tercil intermediário e OR = 12,30; IC95\%: 8,78-17,21, para 1/3 mais pobre) e a chance de dormir com mais de uma pessoa na mesma cama $(\mathrm{OR}=1,31$; $\mathrm{IC} 95 \%$ : 1,01-1,70, para o tercil intermediário e $\mathrm{OR}=2,12$; IC95\%: 1,56-2,88, para as mais pobres).

A exposição positiva do cuidado prestado por uma pessoa diferente da mãe da criança, foi observada tanto para as crianças do tercil intermediário (OR = 0,55; IC95\%: 0,41-0,73) quanto para o mais pobre $(\mathrm{OR}=0,52$; IC95\%: $0,37-0,73)$, quando foi comparada com o tercil das crianças 
Distribuição percentual das características do ambiente doméstico das crianças e de suas famílias, segundo tercis de condições sociais e econômicas em municípios baianos.

\begin{tabular}{|c|c|c|c|c|c|c|c|}
\hline \multirow[t]{2}{*}{ Variável } & \multicolumn{2}{|c|}{$\begin{array}{c}\text { 1o tercil } \\
\text { (mais pobre) }\end{array}$} & \multicolumn{2}{|c|}{$\begin{array}{c}\text { 2o tercil } \\
\text { (intermediário) }\end{array}$} & \multicolumn{2}{|c|}{$\begin{array}{c}\text { 3o tercil } \\
\text { (mais rico) }\end{array}$} & \multirow[t]{2}{*}{$p$ valor } \\
\hline & $\mathrm{n}$ & $\%$ & $\mathrm{n}$ & $\%$ & $\mathrm{n}$ & $\%$ & \\
\hline \multicolumn{8}{|l|}{ Zona de residência } \\
\hline Urbana & 293 & 52,3 & 436 & 75,6 & 551 & 88,3 & \\
\hline Rural & 422 & 47,7 & 202 & 24,4 & 97 & 11,7 & $<0,001$ \\
\hline \multicolumn{8}{|l|}{ Sexo do chefe } \\
\hline Masculino & 515 & 70,2 & 474 & 73,2 & 526 & 82,0 & \\
\hline Feminino & 200 & 29,8 & 164 & 26,8 & 122 & 18,0 & $<0,001$ \\
\hline \multicolumn{8}{|c|}{ Trabalho paterno no momento da entrevista } \\
\hline Empregado & 405 & 53,6 & 391 & 60,0 & 521 & 80,4 & \\
\hline Desempregado & 310 & 46,4 & 247 & 40,0 & 127 & 19,6 & $<0,001$ \\
\hline \multicolumn{8}{|l|}{ Idade materna (anos) } \\
\hline$<20$ & 79 & 11,1 & 79 & 12,6 & 52 & 7,9 & \\
\hline $21-29$ & 340 & 48,6 & 343 & 52,7 & 390 & 60,8 & \\
\hline $30-40$ & 186 & 24,9 & 131 & 21,1 & 157 & 23,5 & $<0,001$ \\
\hline$\geq 40$ & 110 & 15,4 & 85 & 13,6 & 49 & 7,8 & \\
\hline \multicolumn{8}{|c|}{ Anos de estudo materno } \\
\hline > 4a série primária & 111 & 17,0 & 259 & 40,5 & 492 & 76,0 & \\
\hline$\leq$ 4a série primária & 604 & 83,0 & 379 & 59,5 & 156 & 24,0 & $<0,001$ \\
\hline \multicolumn{8}{|l|}{ Criança dorme sozinha } \\
\hline Sim & 183 & 24,6 & 227 & 35,3 & 319 & 49,3 & \\
\hline Não & 532 & 75,4 & 411 & 64,7 & 329 & 50,7 & $<0,001$ \\
\hline \multicolumn{8}{|c|}{ Prestador de cuidado à criança } \\
\hline Mãe & 583 & 82,9 & 515 & 81,4 & 425 & 66,2 & \\
\hline Outro & 132 & 17,1 & 123 & 18,6 & 223 & 33,8 & $<0,001$ \\
\hline \multicolumn{8}{|c|}{ Número de irmãos menores de 5 anos } \\
\hline Um & 614 & 86,7 & 591 & 92,6 & 630 & 97,3 & \\
\hline Mais de um & 101 & 13,3 & 47 & 7,4 & 18 & 2,7 & $<0,001$ \\
\hline
\end{tabular}

mais ricas, mantendo-se assim, o resultado inicialmente observado na análise univariada.

A razão de chance do déficit grave do crescimento linear foi mais elevada para as crianças pertencentes ao tercil mais pobre $(\mathrm{OR}=1,65$; IC95\%: 1,13-2,41), embora ocorresse também entre aquelas do tercil intermediário $(\mathrm{OR}=1,42$; IC95\%: 1,01-2,00).

A ausência do uso de fortificante teve chance de ocorrer tanto para as crianças mais pobres $(\mathrm{OR}=2,13$; IC95\%: 1,49-3,03) quanto para aquelas do tercil intermediário $(\mathrm{OR}=1,35$; IC95\%: $1,01-1,79)$.

Dentre as variáveis do consumo alimentar, somente o retinol mostrou-se associado significantemente às condições de vida das crianças investigadas. Assim, a chance de consumir quantidade do retinol abaixo da mediana é de $50 \%(\mathrm{OR}=1,50$; IC95\%: 1,13-2,00) para as crian- ças do tercil intermediário e aumenta para $86 \%$ $(\mathrm{OR}=1,86$; IC95\%: 1,33-2,60) quando se trata das crianças mais pobres.

Os resultados indicam também que algumas das situações analisadas se associaram somente com as condições de vida situadas no tercil mais pobre, a exemplo da existência de mais de uma criança menor de cinco anos de idade no domicílio (OR = 2,47; IC95\%: 1,15-5,29), ser portador de déficit ponderal leve (OR = 2,93; IC95\%: 1,33$6,45)$, e de moderada/grave intensidades (OR = 1,50; IC95\%: 1,02-2,21), ter o consumo do lipídeo da dieta abaixo da mediana do grupo (OR = 1,55; IC95\%: 1,12-2,16). A ocorrência da anemia só se mostrou associada às condições de vida das crianças do tercil intermediário $(\mathrm{OR}=1,41$; IC95\%: 1,08-1,85), quando comparadas com aquelas do tercil mais rico. 
Odds ratio (OR) bruto para a associação entre o estado nutricional e de saúde das crianças segundo tercis de condições sociais e econômicas em municípios baianos *.

\begin{tabular}{|c|c|c|c|c|c|c|}
\hline \multirow[t]{2}{*}{ Variável } & \multicolumn{3}{|c|}{ 1ㅇ tercil (mais pobre) } & \multicolumn{3}{|c|}{ 2o tercil (intermediário) } \\
\hline & OR & IC95\% & $\mathrm{p}$ valor & OR & IC95\% & $\mathrm{p}$ valor \\
\hline \multicolumn{7}{|l|}{ Indicador altura/idade } \\
\hline Eutrofia & 1,00 & - & - & 1,00 & - & - \\
\hline Déficit leve & 5,07 & $3,15-8,147$ & $<0,001$ & 3,17 & $1,92-5,25$ & $<0,001$ \\
\hline Déficit moderado/grave & 2,38 & $1,82-3,10$ & $<0,001$ & 1,74 & $1,32-2,29$ & $<0,001$ \\
\hline \multicolumn{7}{|l|}{ Indicador peso/idade } \\
\hline Eutrofia & 1,00 & - & - & 1,00 & - & - \\
\hline Déficit leve & 6,32 & $3,56-11,24$ & $<0,001$ & 4,06 & $2,23-7,38$ & $<0,001$ \\
\hline Déficit moderado/grave & 2,20 & $1,70-2,86$ & $<0,001$ & 1,51 & $1,15-1,99$ & 0,003 \\
\hline \multicolumn{7}{|l|}{ Anemia } \\
\hline Não & 1,00 & - & - & 1,00 & - & - \\
\hline $\operatorname{Sim}$ & 1,50 & $1,20-1,87$ & $<0,001$ & 1,59 & $1,27-2,00$ & $<0,001$ \\
\hline \multicolumn{7}{|l|}{ Uso de fortificante } \\
\hline $\operatorname{Sim}$ & 1,00 & - & - & 1,00 & - & - \\
\hline Não & 2,37 & $1,803,12$ & $<0,001$ & 1,37 & $1,06-1,76$ & 0,015 \\
\hline \multicolumn{7}{|l|}{ Historia de morbidade ** } \\
\hline Não & 1,00 & - & - & 1,00 & - & - \\
\hline Sim & 1,27 & $1,01-1,59$ & 0,043 & 1,08 & $0,86-1,37$ & 0,497 \\
\hline \multicolumn{7}{|l|}{ Historia de internamento *** } \\
\hline Não & 1,00 & - & - & 1,00 & - & - \\
\hline $\operatorname{Sim}$ & 1,15 & $0,89-1,50$ & 0,286 & 1,34 & $1,03-1,74$ & 0,030 \\
\hline \multicolumn{7}{|c|}{ Disponibilidade de caloria da dieta } \\
\hline Acima da mediana & 1,00 & - & - & 1,00 & - & - \\
\hline Abaixo da mediana & 2,91 & $2,31-3,66$ & $<0,001$ & 1,77 & $1,41-2,23$ & $<0,001$ \\
\hline \multicolumn{7}{|l|}{ Consumo de proteína } \\
\hline Acima da mediana & 1,00 & - & - & 1,00 & - & - \\
\hline Abaixo da mediana & 2,71 & $2,16-3,41$ & $<0,001$ & 1,77 & $1,41-2,23$ & $<0,001$ \\
\hline \multicolumn{7}{|l|}{ Consumo de lipídio } \\
\hline Acima da mediana & 1,00 & - & - & 1,00 & - & - \\
\hline Abaixo da mediana & 3,27 & $2,60-4,12$ & $<0,001$ & 1,85 & $1,47-2,34$ & $<0,001$ \\
\hline \multicolumn{7}{|l|}{ Consumo de zinco } \\
\hline Acima da mediana & 1,00 & - & - & 1,00 & - & - \\
\hline Abaixo da mediana & 1,44 & $1,15-1,80$ & 0,001 & 1,15 & $0,91-1,44$ & 0,235 \\
\hline \multicolumn{7}{|l|}{ Consumo de retinol } \\
\hline Acima da mediana & 1,00 & - & - & 1,00 & - & - \\
\hline Abaixo da mediana & 2,94 & $2,34-3,70$ & $<0,001$ & 1,91 & $1,52-2,41$ & $<0,001$ \\
\hline \multicolumn{7}{|l|}{ Consumo de ferro } \\
\hline Acima da mediana & 1,00 & - & - & 1,00 & - & - \\
\hline Abaixo da mediana & 2,11 & $1,69-2,65$ & $<0,001$ & 1,58 & $1,26-1,99$ & $<0,001$ \\
\hline
\end{tabular}

* Tomando como referência o 3o tercil (os mais ricos);

** Nos 15 dias anteriores à entrevista;

*** No ano anterior à entrevista.

\section{Discussão}

É reconhecido que em qualquer sociedade a pobreza, enquanto reflexo da apropriação de um rendimento econômico que não é capaz de sa- tisfazer as necessidades mínimas para a sobrevivência da família 4 , associa-se às piores condições de saúde e nutrição. A avaliação da relação entre desigualdade e saúde é um campo de estudo que vem sendo retomado recentemente 
Odds ratio (OR) bruto para a associação entre as variáveis do ambiente doméstico e demográfico das crianças e os tercis de condições sociais e econômicas em municípios baianos *.

\begin{tabular}{|c|c|c|c|c|c|c|}
\hline \multirow[t]{2}{*}{ Variável } & \multicolumn{3}{|c|}{ 1o tercil (mais pobre) } & \multicolumn{3}{|c|}{ 2o tercil (intermediário) } \\
\hline & OR & IC95\% & p valor & OR & IC95\% & $p$-valor \\
\hline \multicolumn{7}{|l|}{ Zona de residência } \\
\hline Urbana & 1,00 & - & - & & & \\
\hline Rural & 6,90 & $5,27-9,02$ & $<0,001$ & 2,44 & $1,85-3,23$ & $<0,001$ \\
\hline \multicolumn{7}{|l|}{ Sexo do chefe } \\
\hline Masculino & 1,00 & - & - & 1,00 & - & - \\
\hline Feminino & 1,93 & $1,48-2,52$ & $<0,001$ & 1,66 & $1,26-2,18$ & $<0,001$ \\
\hline \multicolumn{7}{|c|}{ Trabalho paterno no momento da entrevista } \\
\hline Empregado & 1,00 & - & - & 1,00 & - & - \\
\hline Desempregado & 3,56 & $2,76-4,58$ & $<0,001$ & 2,74 & $2,12-3,55$ & $<0,001$ \\
\hline \multicolumn{7}{|l|}{ Idade materna (anos) } \\
\hline$<20$ & 1,75 & $1,18-2,60$ & 0,005 & 1,84 & $1,25-2,73$ & 0,002 \\
\hline $21-29$ & 1,00 & - & - & 1,00 & - & - \\
\hline $30-40$ & 1,32 & $1,01-1,73$ & 0,041 & 1,03 & $0,78-1,37$ & 0,829 \\
\hline$\geq 40$ & 2,48 & $1,70-3,63$ & $<0,001$ & 2,02 & $1,36-2,99$ & $<0,001$ \\
\hline \multicolumn{7}{|c|}{ Anos de estudo materno } \\
\hline > 4a série primária & 1,00 & - & - & 1,00 & - & - \\
\hline$\leq 4$ a série primária & 15,47 & $11,67-20,50$ & $<0,001$ & 4,64 & $3,62-5,95$ & $<0,001$ \\
\hline \multicolumn{7}{|c|}{ Prestador de cuidado à criança } \\
\hline Mãe & 1,00 & - & - & 1,00 & - & - \\
\hline Outro & 0,40 & $0,311-0,52$ & $<0,001$ & 0,45 & $0,34-0,58$ & $<0,001$ \\
\hline \multicolumn{7}{|l|}{ Criança dorme sozinha } \\
\hline Sim & 1,00 & - & - & 1,00 & - & - \\
\hline Não & 2,99 & $2,36-3,78$ & $<0,001$ & 1,79 & $1,42-2,25$ & $<0,001$ \\
\hline \multicolumn{7}{|c|}{ Número de irmãos menores de 5 anos } \\
\hline Um & 1,00 & - & - & 1,00 & - & - \\
\hline Mais de um & 5,42 & $3,18-9,23$ & $<0,001$ & 2,82 & $1,59-4,99$ & $<0,001$ \\
\hline \multicolumn{7}{|l|}{ Sexo da criança } \\
\hline \multicolumn{7}{|l|}{ Masculino } \\
\hline Feminino & 0,99 & $0,80-1,24$ & 0,958 & 1,15 & $0,91-1,44$ & 0,238 \\
\hline \multicolumn{7}{|c|}{ Idade da criança (meses) } \\
\hline$<6$ & 1,00 & & & 1,00 & & \\
\hline $6-12$ & 1,06 & $0,64-1,59$ & 0,980 & 1,23 & $0,77-2,00$ & \\
\hline $12-24$ & 1,01 & $0,68-1,50$ & 0,952 & 1,22 & $0,81-1,83$ & \\
\hline$\geq 24$ & 0,89 & $0,63-1,25$ & 0,494 & 1,06 & $0,74-1,52$ & \\
\hline
\end{tabular}

* Tomando como referência o 3o tercil (os mais ricos).

em várias partes do mundo 1,25 , voltado principalmente para medir a distância que a riqueza e a pobreza imprimem na eqüidade em saúde tomando-se como referência um determinado patamar de igualdade.

Neste estudo, variáveis da esfera econômica e de acesso/utilização de bens sociais integram um índice que estratificou as crianças e suas famílias em termos de condições de vida, para investigar mediação da relação entre a desigualdade e os fatores do ambiente familiar e condições de saúde e nutrição na infância. Os seus resultados põem em evidência que as desigualdades sociais e econômicas restringem a satisfação das condições básicas de sobrevivência e imprimem marcas indeléveis no estado de saúde e nutrição na infância, desnudando o aumento diferenciado de chance, à proporção que se torna mais precária a condição da inserção familiar no contexto social e econômico. Essa relação já foi detectada em vários países 1, inclusive no Brasil 9,18.

Apesar da melhora dos indicadores de saúde, dos avanços tecnológicos no campo da saúde, da prevenção das doenças por vacinação, do ma- 
Odds ratio (OR) ajustado para a associação entre as variáveis biológicas, do ambiente doméstico e do estado nutricional, segundo os tercis de condições sociais e econômicas em municípios baianos.

\begin{tabular}{|c|c|c|c|c|c|c|}
\hline \multirow[t]{2}{*}{ Variável } & \multicolumn{3}{|c|}{ 1ㅇ tercil (mais pobre) } & \multicolumn{3}{|c|}{ 2o tercil (intermediário) } \\
\hline & OR & IC95\% & $\mathrm{p}$ valor & OR & IC95\% & p valor \\
\hline \multicolumn{7}{|l|}{ Zona de residência } \\
\hline Urbana & 1,00 & - & - & 1,00 & - & - \\
\hline Rural & 9,13 & $6,47-12,90$ & $<0,001$ & 2,83 & $2,06-3,89$ & $<0,001$ \\
\hline \multicolumn{7}{|l|}{ Sexo do chefe } \\
\hline Masculino & 1,00 & - & - & 1,00 & - & - \\
\hline Feminino & 1,88 & $1,29-2,76$ & 0,001 & 1,52 & $1,09-2,12$ & 0,014 \\
\hline \multicolumn{7}{|c|}{ Trabalho paterno no mento da entrevista } \\
\hline Empregado & 1,00 & - & - & 1,00 & - & - \\
\hline Desempregado & 4,90 & $3,47-6,92$ & $<0,001$ & 2,96 & $2,19-4,00$ & $<0,001$ \\
\hline \multicolumn{7}{|l|}{ Anos de estudo materno } \\
\hline > 4a série primária & 1,00 & - & - & 1,00 & - & - \\
\hline$\leq 4$ a série primária & 12,30 & $8,78-17,21$ & $<0,001$ & 4,01 & $3,05-5,27$ & $<0,001$ \\
\hline \multicolumn{7}{|l|}{ Prestador de cuidado à criança } \\
\hline Mãe & 1,00 & - & - & 1,00 & - & - \\
\hline Outro & 0,52 & $0,37-0,73$ & $<0,001$ & 0,55 & $0,41-0,73$ & $<0,001$ \\
\hline \multicolumn{7}{|c|}{ Número de irmãos menores de 5 anos } \\
\hline Um & 1,00 & - & - & 1,00 & - & - \\
\hline Mais de um & 2,47 & $1,15-5,29$ & 0,020 & 1,79 & $0,88-3,66$ & 0,109 \\
\hline \multicolumn{7}{|l|}{ Criança dorme sozinha } \\
\hline Sim & 1,00 & - & - & & & \\
\hline Não & 2,12 & $1,56-2,88$ & $<0,001$ & 1,31 & $1,01-1,70$ & 0,043 \\
\hline \multicolumn{7}{|l|}{ Uso de fortificante } \\
\hline \multicolumn{7}{|l|}{ Sim } \\
\hline Não & 2,13 & $1,49-3,03$ & $<0,001$ & 1,35 & $1,01-1,79$ & 0,044 \\
\hline \multicolumn{7}{|c|}{ Adequação ponderal (peso/idade) } \\
\hline Eutrofia & 1,00 & - & - & 1,00 & - & - \\
\hline Déficit leve & 2,93 & $1,33-6,45$ & 0,008 & 2,06 & $0,98-4,34$ & 0,058 \\
\hline Déficit moderado/grave & 1,50 & $1,02-2,21$ & 0,038 & 1,12 & $0,79-1,57$ & 0,527 \\
\hline \multicolumn{7}{|c|}{ Adequação linear (altura/idade) } \\
\hline Eutrofia & 1,00 & - & - & 1,00 & - & - \\
\hline Déficit leve & 1,81 & $0,90-3,67$ & 0,097 & 1,69 & $0,88-3,23$ & 0,115 \\
\hline Déficit moderado/grave & 1,65 & $1,13-2,41$ & 0,010 & 1,42 & $1,01-2,00$ & 0,042 \\
\hline \multicolumn{7}{|l|}{ Diagnóstico de anemia } \\
\hline Não & 1,00 & - & - & 1,00 & - & - \\
\hline $\operatorname{Sim}$ & 1,20 & $0,88-1,64$ & 0,253 & 1,41 & $1,08-1,85$ & 0,012 \\
\hline \multicolumn{7}{|c|}{ Consumo inadequado de lipídeo } \\
\hline \multicolumn{7}{|c|}{ Não } \\
\hline $\operatorname{Sim}$ & 1,55 & $1,12-2,16$ & 0,009 & 1,18 & $0,89-1,57$ & 0,244 \\
\hline \multicolumn{7}{|l|}{ Consumo de retinol } \\
\hline Acima da mediana & 1,00 & - & - & 1,00 & - & - \\
\hline Abaixo da mediana & 1,86 & $1,33-2,60$ & $<0,001$ & 1,50 & $1,13-2,00$ & 0,006 \\
\hline
\end{tabular}

$N=2001 ; F(38,1962)=13,70, p<0,0001$; ajustado por idade e sexo da criança.

nejo adequado das doenças infecto-contagiosas e das políticas compensatórias, o risco de adoecer e morrer continua mais alto entre as crianças menores de cinco anos de idade das áreas pobres do Brasil, fruto da desigualdade fomentada pela distância entre a riqueza e a pobreza 26 . Esse cenário é observado tanto nas áreas urbanas quanto no espaço rural do país. No entanto, o diferencial entre o urbano e o rural se faz sentir com toda a sua força quando se trata das condições 
materiais de vida, saúde e nutrição das crianças no Nordeste brasileiro.

Neste sentido, a chance da criança da zona rural pertencer ao estrato mais pobre é maior do que a encontrada para a criança residente no espaço urbano e, diminui quando se trata da inclusão da criança do estrato intermediário. Esses resultados são consistentes com as informações disponíveis para o Nordeste rural, onde são registradas as taxas mais elevadas de mortalidade infantil, da desnutrição energético protéica e da inadequação dos indicadores sociais 5 .

No cenário da desigualdade social, observouse que ser cuidada por outra pessoa diferente da genitora constituiu-se em exposição protetora de expressão similar para as crianças que vivem nos dois tercis mais baixos das condições de vida. A leitura desse resultado não permite concluir pela ação nefasta do cuidado materno para a criança; desafortunadamente as informações disponíveis neste estudo não permitem conhecer o motivo pelo qual a mãe se ausentava do domicílio, mas, permite pressupor uma relação compensatória entre a participação da mulher no mercado de trabalho e assim, mantenedora de bens materiais na unidade familiar e, por conseguinte, da saúde da criança 27. Essa suposição se fortalece no fato de que as chances de desemprego paterno e o exercício da chefia da residência pela mulher estão presentes nos dois tercis mais precários das condições de vida e são mais elevadas entre as crianças mais pobres. Nesses patamares de vida social e econômica, a ausência materna, possivelmente por motivo de trabalho, pode contribuir com o aumento da renda e prover as necessidades básicas da unidade familiar, traduzindo-se em proteção 28 .

No leque da expressão da desigualdade nos municípios amostrados, observou-se que a prevalência da desnutrição leve e moderada/grave, medida tanto pelo indicador peso/idade quanto altura/idade, é mais elevada entre as crianças do estrato mais pobre diminuindo à proporção que a criança se desloca do tercil menos pobre para o mais rico, com um nítido efeito "dose resposta”.

No entanto, observa-se que o tercil intermediário das condições de vida não imprime risco ao crescimento ponderal da criança, o que ocorre somente quando estas são as mais pobres. Esse mesmo diferencial não foi observado para o crescimento linear, dado que as crianças dos dois tercis de condições de vida menos favoráveis têm chances crescentes e estatisticamente significantes de déficits moderado/grave do indicador altura/idade, sinalizando que pertencer ao tercil intermediário não elimina a chance de déficit no crescimento linear, possivelmente porque este indicador é extremamente sensível às condições inadequadas do ambiente social e econômico ${ }^{3}$.

A expressão das condições desfavoráveis do ambiente doméstico é dada também pela aglomeração domiciliar. As crianças do tercil intermediário e as mais pobres têm maiores chances de dividirem o espaço de dormir com outra pessoa. E as famílias mais pobres têm chances mais elevadas de contar com mais de uma criança menor de cinco anos de idade no domicílio, quando comparadas com as mais ricas. Além dos efeitos dessa situação na divisão intradomiciliar do alimento e dos escassos recursos domésticos, promovem também o compartilhamento da mesma cama para dormir, com prejuízos para o relaxamento corporal, o que contribui para restringir o crescimento linear das crianças, uma vez que as evidências indicam que a liberação do hormônio do crescimento é maior à noite, durante o sono, quando as condições de relaxamento corporal são adequadas 29 .

O contexto social e econômico identificado para as crianças investigadas explica também o inadequado acesso aos alimentos que se expressa no consumo de lipídio abaixo da mediana para as crianças mais pobres e de retinol para $50 \%$ das crianças do tercil intermediário e para as mais pobres (Tabela 4). As evidências indicam que a disponibilidade de gordura na dieta da criança no Nordeste brasileiro não alcança o porcentual de $30 \%$ recomendado 30 . É conhecido o efeito constrangedor do baixo teor do lipídeo na conversão dos carotenóides em retinol 31 . Esse fato merece destaque particularmente porque os carotenóides são as principais fontes de vitamina A das dietas das crianças em várias regiões do Nordeste brasileiro 12,32 e, as evidências indicam que o consumo inadequado de vitamina A se associa com o aumento significante do risco de mortalidade 33 e morbidade, especialmente pelas diarréias 34. Essa condição tem sido minimizada quando a operacionalização do Programa de Suplementação com Vitamina A 35 ocorre de maneira sistemática e continuada.

É consensual que prevalências de anemia maiores que $40 \%$ têm como causa mais provável a deficiência de ferro dietético ${ }^{16}$. Entre as crianças investigadas a anemia acometia $48,2 \%$ delas (Tabela 1). No entanto, a associação significante entre a anemia e as condições de vida, previamente observada na análise univariada para as crianças do estrato de vida intermediário e do mais pobre, persistiu somente para aquelas do tercil intermediário na análise multivariada. Esse resultado pode indicar que outros eventos estejam protegendo essas crianças e impedindo que essa associação assuma também a importância 
estatística esperada entre as crianças do estrato de condições de vida mais precário.

Assim, os resultados deste estudo põem em evidência a associação entre a desigualdade social e econômica e situações diferenciadas de acesso aos alimentos, das precárias condições do ambiente familiar e do estado de saúde e nutrição na infância, e torna possível compreender que somente a melhoria na qualidade de vida da população pode fomentar a diminuição da pobreza e da desigualdade, e garantir a eqüidade e a inclusão social. Nesse sentido, a distribuição justa da riqueza socialmente produzida é um pressuposto básico da inclusão social e econômica e da eqüidade em saúde e nutrição. Mas entende-se também que as políticas de saúde podem diminuir a desigualdade, entendida aqui como destacada por Minayo 4: manifestação das distâncias relativas entre os estratos populacionais que se expressa em condições diferenciadas na perspectiva da apropriação da riqueza produzida, dos bens e serviços, direitos sociais e políticos de um país.
Assim, o modelo análitico adotado neste estudo, permitiu avaliar não somente a associação entre a iniqüidade social e as condições de saúde nos extremos das condições de vida das crianças investigadas, mas considerar também essas relações para o grupo intermédiário, o que normalmente não é visto nos estudos que abordam essa problemática. Ressalta-se no entanto, que mesmo adotando todos os pressupostos metodológicos para a plicação da técnica logística multinomial e a ponderação para o efeito do conglomerado, o modelo transversal adotado neste estudo impede traçar relação causal entre desigualdade e condições diferenciadas de vida e saúde.

Por último cabe comentar, que dada a complexidade do processo amostral adotado neste estudo, conglomerado em três estágios, envolvendo o município, o domicílio e a criança, a análise multinível seria a mais recomendada 36 No entanto, o estágio do conhecimento ainda não permite a aplicação da abordagem multinível com a estratificação da variável dependente em três níveis, como no desenho analítico adotado neste estudo.

\section{Resumo}

Estudo transversal, envolvendo 2.001 crianças das áreas rural e urbana de dez municípios da Bahia, Brasil. Avalia-se a relação entre os gradientes da desigualdade e os fatores do ambiente familiar, de saúde e nutrição, utilizando-se a regressão logística multinomial multivariada. As crianças do tercil intermediário das condições de vida e aquelas do tercil mais pobre têm chances significantes e crescentes de viverem na área rural, em domicílio chefiado pela mulher, ter o pai desempregado, mãe com baixa escolaridade, de dormir com mais de uma pessoa na mesma cama, déficit linear grave e consumir retinol abaixo da mediana. A existência de mais de uma criança no domicílio, ser portadora de déficit ponderal e ter o consumo de lipídeo abaixo da mediana foram eventos também significantes para as crianças mais pobres. A anemia foi identificada entre as crianças do tercil intermediário. A desigualdade expõe as crianças a chances crescentes de inadequado estado de saúde e nutrição. Políticas de saúde podem implementar medidas emergenciais no sentido de minimizar os males impostos pela desigualdade à saúde e nutrição na infância.

Iniquidade Social; Fatores Socioeconômicos; Bem-Estar da Criança

\section{Colaboradores}

A. M. O. Assis foi responsável pelo desenho, supervisionou o trabalho de campo, coordenou a análise e elaboração final do manuscrito. M. L. Barreto foi responsável pelo desenho, coordenação e elaboração final do manuscrito. N. S. Santos coordenou o trabalho de campo, foi responsável pela análise de consumo alimentar e contribuiu com a elaboração final do manuscrito. L. P. M. Oliveira coordenou o trabalho de campo, foi responsável pela análise de consumo alimentar e contribuiu com a elaboração final do manuscrito. S. M. C. Santos foi responsável pela elaboração final do manuscrito. S. M. C. Pinheiro foi responsável pelas análises estatísticas e interpretação dos resultados, contribuiu na elaboração final do manuscrito.

\section{Agradecimentos}

Estudo patrocinado pela Financiadora de Estudos e Projetos (FINEP; convênio 64.96.0564.00) e pelo Ministério da Saúde/Centro Colaborador Nordeste II (convênio 567/99) 


\section{Referências}

1. Blakely T, Hales S, Kieft C, Wilson N, Woodward A. The global distribution of risk factors by poverty level. Bull World Health Organ 2005; 83:118-26.

2. Makinen $\mathrm{M}$, Waters $\mathrm{H}$, Rauch $\mathrm{M}$, Almagambetova $\mathrm{N}$, Bitran R, Gilson L, et al. Inequalities in health care use and expenditures: empirical data from eight developing countries and countries in transition. Bull World Health Organ 2000; 78:55-65.

3. United Nations Administrative Committee on Coordination/Sub-Committee on Nutrition. Nutrition and poverty. Geneva: United Nations Administrative Committee on Coordination/Sub-Committee on Nutrition, 1997. (Report Nutrition Policy Paper, 161997).

4. Minayo MCS. Condiciones de vida, desigualdad y salud a partir del caso brasileño. In: Briceño-León R, Minayo MCS, Coimbra Jr. CEA, editores. Salud y equidad: una mirada desde las ciencias sociales. Rio de Janeiro: Editora Fiocruz; 2000. p. 55-72.

5. Instituto Brasileiro de Geografia e Estatística. In dicadores sociais. http://www.ibge.gov.br/home/ estatistica/populacao/condicaodevida/ indicadoresminimos/sinteseindicsociais2004/ indic_sociais (acessado em 17/Abr/2004).

6. Sociedade Civil Bem-Estar Familiar no Brasil/ Demographic and Health Survey. Pesquisa Nacional sobre Demografia e Saúde 1996. 2a Ed. Rio de Janeiro: Sociedade Civil Bem-Estar Familiar no Brasil/Demographic and Health Survey; 1999.

7. Instituto Brasileiro de Geografia e Estatística. Pesquisa de orçamentos familiares - 2002-2003 (POF): estado antropométrico infantil. http://www.ibge. gov.br/home/estatistica/populacao/condicaodevida/pof/2003medidas/default.shtm (acessado em 22/Dez/2006)

8. Departamento de Atenção Básica, Secretaria de Atenção à Saúde, Ministério da Saúde. Avaliação do Programa Bolsa-Alimentação: primeira fase. Brasília: Ministério da Saúde; 2005. (Série C. Projetos, Programas e Relatórios).

9. Drachler ML, Côrtes SMV, Castro JD, Leite JCC. Proposta de metodologia para selecionar indicadores de desigualdade em saúde visando definir prioridades de políticas públicas no Brasil. Ciênc Saúde Coletiva 2003; 8:461-70.

10. Barreto ML, Oliveira LPM, Assis AMO, Braga-Junior ACR, Nunes MFP, Oliveira NF. Indicadores socioeconômicos no diagnóstico e planejamento em saúde e nutrição. Brasília: Financiadora de Estudos e Projeto; 2002.

11. Instituto Brasileiro de Geografia e Estatística. Censo demográfico 1991: resultado do universo relativo às características da população e dos domicílios, Bahia, $n^{\circ}$. 17. Rio de Janeiro: Instituto Brasileiro de Geografia e Estatística; 1994.

12. Assis AMO, Barreto ML, Santos LMP, Sampaio LR, Magalhães LP, Prado MS, et al. Condições de vida, saúde e nutrição na infância em Salvador. Salvador: Editora Bureau; 2000.

13. Siqueira AL, Sakurai E, Souza MCFM. Dimensionamento de amostras em estudos clínicos e epidemiológicos. Salvador: Associação Brasileira de Estatística; 2001.
14. Lohman TG, Roche AF, Martorell R. Anthropometric standardization reference manual. Champaign: Human Kinetics Books; 1988.

15. Organización Mundial de la Salud. Medición del cambio del estado nutricional. Geneva: Organización Mundial de la Salud; 1983.

16. World Health Organization. Iron deficiency anaemia: assessment, prevention and control. Geneva: World Health Organization; 2001.

17. Magalhães LP, Oliveira VA, Santos JM. Guia para estimar consumo alimentar. Salvador: Núcleo de Pesquisa de Nutrição e Epidemiologia, Escola de Nutrição, Universidade Federal da Bahia; 1996.

18. Isller RMS, Giugliani ERJ. Identificação de grupos mais vulneráveis à desnutrição infantil pela medição do nível de pobreza. J Pediatr (Rio de J) 1997; 73:101-5.

19. Alvarez ML, Wurgaft F, Salazar ME. Measurements of the urban low socioeconomic level in families with malnourished infants. Arch Latinoam Nutr 1982; 32:650-62.

20. Hamill PV, Drizd TA, Johnson CL, Reed RB, Roche AF. NCHS growth curves for children birth-18 years. United States. Vital Health Stat 11 1977; (165):i-iv, 1-74.

21. Pelletier DL. The relationship between child anthropometry and mortality in developing countries: implications for policy, programs and future research. J Nutr 1994; 124 Suppl 1:2047-81.

22. Wintrobe M, Lee GR, Bogs TR, Bithell TC, Foerster J, Athens J, et al. Clinical hematology. Philadelphia: Lea \& Febiger; 1981.

23. Wickens TD. Categorical data analysis. Rev Psychol 1998; 48:537-58.

24. Hosmer DW, Lemeshow S. Applied logistic regression. New York: Jonh Wiley and Sons; 1989.

25. Regidora E, Gutierrez-Fisac JL, Dominguez V, Calle ME, Navarro P. Comparing social inequalities in health in Spain 1987 and 1995/97. Soc Sci Med 2002; 54:1323-32.

26. Duarte EC, Schneider MC, Paes-Souza R, Ramalho WM, Sardinha LMV, Silva Júnior JB, et al. Epidemiologia das desigualdades em saúde no Brasil: um estudo exploratório. http://www.funasa.gov. $\mathrm{Br} /$ pub/epi_desigualdades (acessado em 22/ Jun/2003).

27. The State of the World's Children 1998: a UNICEF report. Malnutrition: causes, consequences, and solutions. Nutr Rev 1998; 56:115-23.

28. Facchini LA. Trabalho materno e ganho de peso infantil. Pelotas: UFPEL Editora e Gráfica; 1995.

29. Evain-Brion D, Fjellestad-Paulsen A, Czernichow $\mathrm{P}$, Porquet D, Therond P, Grenèche M-O, et al. Vitamin A deficiency and nocturnal growth hormone secretion in short children. Lancet 1994; 343:87-8.

30. World Health Organization. Complementary feeding of children in development countries: a review of current scientific knowledge. Geneva: World Health Organization; 1998. (Technical Report WHO/NUT/98.1). 
31. Underwood BA, Chavez M, Hankin J. Guidelines for the development of a simplified dietary assessment to identify groups at risk for inadequate of vitamin A. Geneva: International Vitamin A Consultative Group; 1989.

32. Prado MS, Assis AMO, Freitas MCS, Silva RCR, Varjão ML. Padrão e seleção de alimentos complementares e sucedâneos do leite materno em comunidades rurais no semi-árido baiano. Rev Nutr PUCCAMP 1995; 8:47-64.

33. Beaton GH, Martorell R, L'Abbé KL, Edmonston B, McCabe G, Ross AC, et al. Effectiveness of vitamin A supplementation in the control of young child morbidity and mortality in developing countries. SCN News 1993; 9:17-21.
34. Barreto ML, Santos LMP, Assis AMO, Araújo MPN Farenza GG, Santos PAB, et al. Effect of vitamin A. Supplementation on diarrhea and acute lower-respiratory-tract infections in young children in Brazil. Lancet 1994; 344:228-31.

35. Brasil. Portaria do Gabinete do Ministro de Estado da Saúde de no. 2.160, de 29 de dezembro de 1994, que aprova as diretrizes e as estratégias do Programa Nacional de Controle das Deficiências de Vitamina A. Diário Oficial da União 1994; $30 \mathrm{dez}$.

36. Diez-Roux AV. Multilevel analysis in public health research. Annu Rev Public Health 2000; 21:171-92.

Recebido em 24/Mar/2006

Versão final reapresentada em 23/Fev/2007

Aprovado em 04/Abr/2007 